\title{
Fitter in-body bioelectronics
}

\author{
Implantable sensors and electrodes that take advantage of new materials, device designs and fabrication strategies \\ enable new and improved biomedical applications.
}

S oft, biocompatible, resorbable, flexible, minimally invasive, durable, batteryless and enabling wireless data transfer; these are just a few of the key requirements for state-of-the-art bioelectronic devices for implantation in the body. Sensors and electrodes that mechanically behave like the tissue they are embedded into (or adhered to) cause less tissue damage, make better tissue/device interfaces and trigger weaker immune responses (traditional metal electrodes typically get surrounded by scar tissue, impairing their function). Furthermore, biocompatible and fully resorbable implanted devices do not need to be extracted after they are no longer functional; they degrade, and their components and by-products are assimilated by the body or excreted from it. Flexible implantable devices better accommodate body movements, and can be stretched, compressed or folded before implantation, so that they can be delivered more easily, for example via a needle or a small catheter. Bioelectronic devices that are stable when in contact with biofluids will also have longer operational lifetimes. Moreover, battery-less and wireless bioelectronic devices, although constrained by the amount of power and data that can be transferred, can be miniaturized further (batteries that provide power to sensory and stimulatory functions can be bulky and not biocompatible), placing less constraints on body motion and device implantability.

Unsurprisingly, it is difficult to design and fabricate sensors or electrodes that fit all the general requirements of an ideal bioelectronic device for body implantation. Yet, as with most engineering problems, feasibility often lies in determining the right balance of requirements and constraints. For example, metals are not intrinsically compatible with tissue, do not efficiently convert changes in ionic charge to electronic signals, and their function as electronic components can be impaired when in direct contact with biofluids; yet metals are durable, stable and amenable to standard fabrication processes. Also, most polymers (in particular, polymer gels) do not conduct electricity well and may require bespoke synthesis or microfabrication methods, yet they can be easily functionalized and made to be compliant, transparent and resorbable.

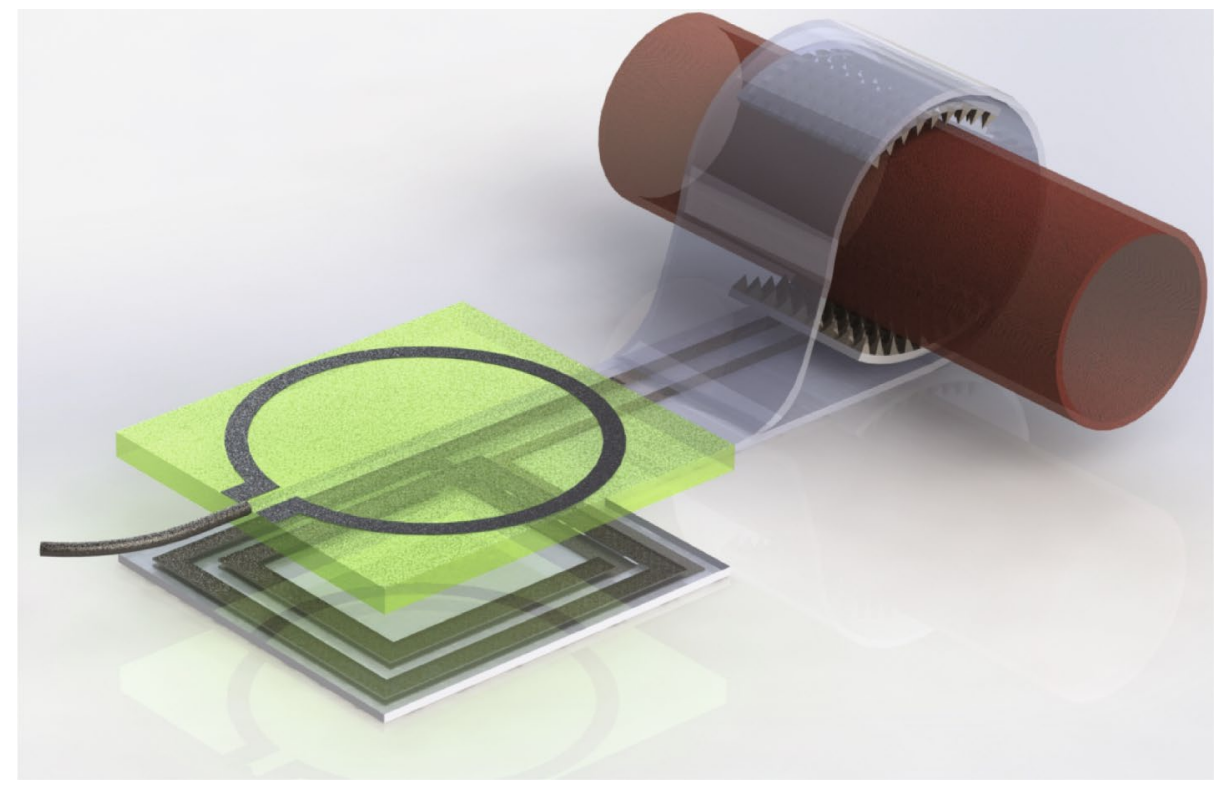

A biodegradable, flexible, battery-free and wireless implantable sensor for monitoring blood flow in an artery. An external reader coil (green plane) captures, via inductive coupling, changes in the capacitance of the sensor resulting from artery expansion and contraction. Credit: Image reproduced from Boutry et al., Springer Nature, Ltd

In this respect, the six research articles included in this issue exemplify the need to balance functionalities in the presence of biological, materials and engineering constraints by using new device designs, new materials or new fabrication strategies to make sensing or stimulating implantable devices for specific biomedical applications.

Wilson Ray, John Rogers and colleagues made silicon-based pressure sensors, shaped as a flat 1-mm square and protected from biofluids by an ultrathin silica coating, that completely resorb after their operational lifetime (which depends on the thickness of the coating) by transforming into watersoluble products. When implanted in the brain of rats, the sensors could accurately monitor intracranial pressure for over 25 days, with no evidence of toxicity and immune responses. Paige Fox, Zhenan Bao and co-authors also developed a pressure sensor that is resorbed by the body long after implantation. Because its intended use was to monitor blood flow in small arteries (less than $2 \mathrm{~mm}$ in diameter), the cuff-like device had to be designed so that it does not damage the artery and can measure changes in its diameter (even when not in contact) via capacitance changes (in the fringe field). By taking advantage of inductive coupling, the authors made the implantable device (pictured) wireless and battery-free.

Zhenan Bao's team also designed a microelectrode array made of conductive porous hydrogel (encapsulated in elastic insulating polymer) that is stable in water and wraps around a peripheral nerve to electrically stimulate it at low voltage. The hydrogel's stretchability helped maintain a stable device-nerve interface and minimized immune responses against the device. When wrapping the sciatic nerve of mice, the device triggered muscle responses at a voltage that was ten-times lower than that of conventional platinum electrodes. Stretchability is also necessary when a device needs to stay adhered to tissue. Yuji Morimoto, Toshinori Fujie and colleagues developed ultrathin polymer sheets modified with a bioadhesive to stably fix to the inner surface of a mouse's skin a wirelessly powered optoelectronic device, 
sandwiched between the polymer sheets, that delivered light to an intradermally transplanted tumour, to perform low-dose long-term photodynamic therapy.

Bioelectronic devices that use readily available components can be customized to enable new applications. Rikky Muller and colleagues developed a 128-channel wireless device incorporating on-board electronics to record, with real-time artefact cancellation, and modulate (after computation of appropriate biomarkers based on the recorded local field potentials) neural activity in the brain of a non-human primate to change its motor behaviour while carrying out a joystick task. And Alan Jasanoff and colleagues designed wireless and batteryless inductively coupled resonant circuits, $3 \mathrm{~mm}$ in diameter, that change their properties in response to the electrical activity or bioluminescent signals of surrounding cells, transducing them to signals that can be detected by an MRI scanner. When implanted in the brain of rats alongside luciferase-expressing cells, the transducers enabled the detection of the bioluminescence produced by the cells.

These proof-of-concept wireless and resorbable pressure sensors, soft nerve stimulators, and miniaturized devices with new functionality highlight the evolution of materials, designs and fabrication methods to make bioelectronic devices that will eventually be fit for the human body.

Published online: 8 January 2019

https://doi.org/10.1038/s41551-018-0346-3 